\title{
The acylation-stimulating protein pathway and regulation of postprandial metabolism
}

\author{
BY ALLAN D. SNIDERMAN ${ }^{1}$, KATHERINE CIANFLONE ${ }^{1}$, LUCINDA SUMMERS $^{2}$, \\ BARBARA FIELDING ${ }^{2}$ AND KEITH FRAYN ${ }^{2}$ \\ 'McGill Unit for the Prevention of Cardiovascular Disease, Royal Victoria Hospital, \\ 687 Pine Avenue West, Montreal, Quebec H3A IA1, Canada \\ ${ }^{2}$ Oxford Lipid Metabolism Group, Radcliffe Infirmary, Oxford OX2 $6 H E$
}

By regulating the rate of adipocyte triacylglycerol synthesis, the acylation-stimulating protein (ASP) pathway plays a critical role in postprandial triacylglycerol clearance (Baldo et al. 1993). The objective of the present paper is to outline how the ASP pathway operates and to describe the temporal relationship between chylomicron triacylglycerol hydrolysis and ASP generation by adipose tissue in vivo. If these observations are taken together with the previous in vivo studies of subcutaneous adipose tissue metabolism by Frayn et al. $(1994 a, b)$, substantial and fascinating differences in the metabolism of VLDL and chylomicrons become evident. Most importantly, appreciation of the role of the ASP pathway allows a critical distinction to be drawn between the overall rate of triacylglycerol clearance from plasma and the effectiveness of triacylglycerol storage in adipose tissue, it being dysfunction of the latter process which is intimately linked with the overproduction of hepatic apolipoprotein B-100 lipoproteins so commonly found in patients with coronary artery disease.

\section{THE ASP PATHWAY: IN VITRO DATA}

ASP is produced by the interaction of three proteins which have been shown to be secreted by both murine and human adipocytes. These are the third component of complement (C3), factor B, and adipsin (or factor D). These three proteins interact such that a seventy-seven amino acid amino terminal fragment of $\mathrm{C} 3$ is generated. In vivo, however, the terminal arginine of this fragment is removed almost immediately by carboxypeptidases to produce a seventy-six amino acid basic peptide, ASP. By a variety of techniques, however, we have shown that ASP is identical to C3adesarg, the terminal product of the alternate complement pathway (Baldo et al. 1993).

ASP is the most potent stimulant of triacylglycerol synthesis in human adipocytes yet described (Cianflone et al. 1989a,b). ASP also markedly increases triacylglycerol synthesis in human skin fibroblasts (Cianflone et al. 1989a,b), but it produces only a relatively modest stimulation of triacylglycerol synthesis in HepG2 cells (Cianflone et al. 1990b). ASP is produced in differentiated human adipocytes to a much greater extent than in human pre-adipocytes, and the increase in ASP production during differentiation is associated with increased $\mathrm{mRNA}$ for $\mathrm{C} 3$, adipsin, and factor $\mathrm{B}$, increases which precede the marked increase in triacylglycerol synthetic capacity which occurs in these cells as they differentiate (Cianflone et al. 1994; Cianflone \& Maslowska, 1995). Of particular importance, as adipocytes differentiate and become larger with greater triacylglycerol synthetic capacity and triacylglycerol mass, they become more responsive to ASP (Cianflone et al. 1995); i.e. in contrast with the response to insulin, larger adipocytes 
display no evidence of resistance to the stimulatory effects of ASP on triacylglycerol synthesis (Walsh et al. 1989).

The mechanisms by which ASP causes triacylglycerol synthesis to increase are now known in some detail. Two different effects can be demonstrated: the first being stimulation of the last (in adipose tissue probably rate-limiting) enzyme (Haagsman et al. 1982) involved in triacylglycerol synthesis, diacylglycerol acyltransferase (Yasruel et al. 1991); the second, an increase in specific membrane transport of glucose (Germinario et al. 1993; Maslowska et al. 1997; Tao et al. 1997). Increased diacylglycerol acyltransferase (EC 2.3.1.20) activity produces increased fatty acid incorporation into triacylglycerol and, therefore, an increased rate of fatty acid uptake by adipocytes. The other effect of ASP, the stimulation of specific membrane glucose transport, is also of considerable interest. ASP stimulates glucose transport in human skin fibroblasts (Germinario et al. 1993), human adipocytes (Maslowska et al. 1997), and L6 myotubes (Tao et al. 1997). Of interest is the observation that the effects of ASP on glucose transport appear to be independent of insulin and, in the case of human skin fibroblasts and L6 myotubes, additive to insulin.

\section{THE ASP PATHWAY: IN VIVO DATA}

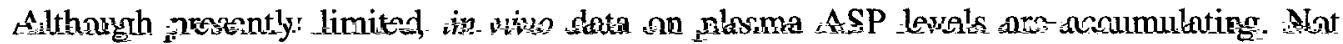
unexpectedly, plasma ASP levels are elevated in obesity and return towards: normal with weight loss (Sniderman et al. 1991; Cianflone et al. 1995). Plasma ASP levels are increased in patients with coronary artery disease (Cianflone et al. 1997) and the potential significance of this will be discussed later (p. 710). At this point, however, it is the postprandial data on which we will focus. In normal subjects following an oral glucose load, plasma ASP does not change, while following an oral fat load, plasma ASP levels gradually rise and then fall, the changes closely linked in time with the changes in plasma triacylglycerol (Cianflone et al. 1989b). If ASP is involved in triacylglycerol clearance, a significant increase in the postprandial period would be anticipated.

These data raised the possibility that chylomicrons might be involved in the processes which lead to the increased generation of ASP, and the relationships between the generation of ASP in the postprandial period and chylomicron metabolism have been pursued further. In a recently completed study, plasma samples were obtained from a superficial anterior abdominal wall vein which drains subcutaneous adipose tissue, and samples of arterialized venous blood were also obtained from a vein draining the hand (J. Saleh, K. Cianflone, A. D. Sniderman, L. Summers and K. Frayn, unpublished results). Matched sets of samples were obtained during an oral fat load administered to eight normal and four obese subjects and the veno-arterial gradients for plasma triacylglycerol and ASP were calculated.

As expected, there is a negative veno-arterial gradient for triacylglycerols, pointing to removal of triacylglycerols from plasma by the adipose tissue as the blood passes through. By contrast with triacylglycerols, the veno-arterial gradient for ASP is positive, establishing that ASP is generated within the adipose tissue microenvironment in the postprandial period, a portion of that which has been generated escaping in the venous effluent to enter the general circulation. However, the temporal relationship between the removal of triacylglycerols and the production of ASP is also of considerable interest, with the most marked removal of triacylglycerols occurring when the generation of ASP has become substantial within the adipose tissue bed. The temporal relationship between the plasma triacylglycerol curve and the veno-arterial triacylglycerol differences deserves comment as well. During the first half of the postprandial period, plasma triacylglycerols 
rise, indicating that the removal capacity has been exceeded. But, as previously noted, the veno-arterial gradients indicate that maximal removal of triacylglycerols does not occur until the second half of the postprandial period. This paradox can only be resolved if the removal capacity varies during the postprandial period, being substantially less in the first half compared with the second half of the period. By increasing the adipocyte triacylglycerol synthetic capacity, ASP should increase triacylglycerol removal by adipose tissue. Since ASP release is maximal in the second half of the postprandial period, greater triacylglycerol removal by adipose tissue during this time would be expected.

These findings are consistent, therefore, with the hypothesis that ASP does play an important role in the uptake of fatty acids from chylomicrons to adipocytes. They also suggest a causal relationship between chylomicrons and generation of ASP. That such a relationship exists has been demonstrated during in vitro experiments which sought to determine which factor(s) added to the medium in which human adipocytes were cultured caused increased generation of ASP (Maslowska et al. 1997). Glucose and fatty acids had no effect, whereas insulin caused a modest (two-to-threefold) but significant increase in ASP generation. By contrast, chylomicrons caused a profound increase in ASP generation when added to the medium, the effect being time- and concentration-dependent. The other lipoproteins, VLDL, LDL, and HDL, had no stimulatory effect. The mechanism by which chylomicrons produce the effect is now being studied in detail, but involves greatly increased secretion of $\mathrm{C} 3$ by adipocytes, thus the increase in ASP is not simply due to enhanced interaction of its three precursor proteins and increased conversion of C3 to ASP.

\section{DIFFERENCES IN CHYLOMICRON AND VLDL METABOLISM BY ADIPOSE TISSUE}

These new findings must be added to those already obtained which indicate marked differences in the metabolism of VLDL and chylomicrons by adipose tissue. Until recently, only quantitative differences between the metabolism of chylomicrons and VLDL by peripheral cells were appreciated. Both lipoproteins are triacylglycerol-rich and both are acted on by lipoprotein lipase (EC 3.1.1.34; LPL) which has been activated by apo C-II. In vivo chylomicron triacylglycerols are certainly hydrolysed much more rapidly than VLDLtriacylglycerols (Grundy \& Mok, 1976), but the underlying mechanisms that explain the degree of this difference have yet to be established in vitro.

However, recent in vivo studies in human subjects of the metabolism of these lipoproteins have added much new, and in some respects unanticipated, information. One of the most important of these observations is that, with the exception of the very largest particles, VLDL are degraded sequentially, larger particles being converted into smaller ones (Packard et al. 1986). Because sequential degradation can be recognized in plasma, it can be concluded that VLDL particles bind to endothelial cells, the triacylglycerols within them are partially hydrolysed, and the particles are then released again into the general circulation. They bind again and are released again. The number of times this sequence is repeated is unknown, but that such a sequence exists seems certain from the radiotracer data which show precursor-product relationships between large and smaller VLDL particles which become in turn IDL and LDL particles.

The peripheral metabolism of chylomicrons and very large VLDL differ from this model. In normal subjects, postprandially, no more than one-third of the particles in the Svedberg flotation unit $\left(S_{f}\right)>400$ fraction are converted to $S_{f} 60-400$ material and, equally strikingly, no further conversion to denser particles can be recognized (Karpe et al. 1995). Given their short half-life in plasma and given the time required for LPL to hydrolyse the 
triacylglycerols within them, chylomicrons seem likely to bind only once to endothelial cells before being removed irreversibly from the circulation.

Why then are chylomicrons cleared so much more rapidly than VLDL? Goldberg (1996) has pointed out that since, on average, VLDL are one-fifth the diameter of chylomicrons, they have one-twenty-fifth the chance of contacting the capillary endothelium compared with chylomicrons. On the other hand, VLDL particles outnumber chylomicron particles by at least 20:1 (Karpe et al. 1995), so that this explanation, i.e. greater propensity to initial contact, seems unlikely to hold. More probably, we believe, there is a difference in the strength of association once formed. Chylomicrons almost certainly will interact with many more LPL molecules than will VLDL and the overall strength of association will be correspondingly greater. Moreover, the strength of association between the triacylglycerol-rich lipoproteins and the endothelial surface will be determined by the rate at which fatty acids can be taken up by the tissue. This transfer or trapping of fatty acids is much greater in the case of chylomicrons than VLDL. The factors which determine this transfer rate (surface area of contact and activation of the ASP pathway) will be explained later.

The second major difference in the peripheral metabolism of VLDL and chylomicrons has been shown by the studies of subcutaneous adipose tissue metabolism by Frayn et al. $(1994 a, b)$, and concerns the difference in the fate of the fatty acids which have been released from them. In the case of chylomicrons, about $50 \%$ of the fatty acids which are released are taken up by the adipocytes within the microenvironment, whereas in the case of VLDL, the percentage taken up is very much less, being only about $5 \%$ (Frayn et al. $1994 a, b)$. These differences may explain the previous statement, i.e. that there may be major differences in the endothelial binding characteristics of the two triacylglycerol-rich lipoproteins. Chylomicrons are much larger than VLDL and so the surface contact with the endothelium should be much larger. Scow et al. (1976) have examined the physical pathway by which fatty acids must move to adipocytes and, simply put, fatty acids must move through endothelial cell membranes, within and through the endothelial cell to reach the underlying adipocytes. Could the much greater trapping of fatty acids released from chylomicrons be due, at least in part, to the greater surface interaction per bound particle compared with VLDL? The greater the bound surface, the more intense the binding to the endothelial cells and the greater the area available for fatty acids to exit to adipocytes rather than escape to the plasma compartment. In addition, however, we believe there is another explanation for the difference between the peripheral metabolism of chylomicrons and VLDL; i.e. the effectiveness of the ASP pathway in trapping of fatty acids released from chylomicrons.

\section{THE ASP PATHWAY AS A DETERMINANT OF TISSUE TRAPPING OF FATTY ACIDS RELEASED FROM CHYLOMICRONS}

The overall rate of plasma triacylglycerol clearance is the sum of several processes. The first of these is the rate at which chylomicrons are able to bind to endothelial cells within tissues such as adipose tissue and muscle, and be hydrolysed by LPL. Until the action of LPL on a bound particle is completed, no other particle can take its place. However, that is not the whole story; also crucial is what happens to the fatty acids which are released. Once LPL starts to act, fatty acids are released suddenly and massively within the capillary microenvironment. If these fatty acids are not immediately transferred to the adipocytes in the subendothelial space, they will bind to albumin, leave the capillary, and enter the 
general circulation (Fig. 1). There are thus two paths they may take: one to the adipocyte, the other to the general circulation.

The ASP pathway, by influencing the rate of adipocyte triacylglycerol synthesis, is critical in determining which route the newly liberated fatty acids actually take. To the extent that adipocyte triacylglycerol synthesis increases as required, then fatty acids released from chylomicrons will be directed into these cells and not released into the general circulation. There is, therefore, necessarily an inverse relationship between these two metabolic fates; the greater the proportion of fatty acids that are trapped by the adipocytes, the smaller the proportion that will enter the general circulation. In this model, the ASP pathway promotes energy storage by increasing the proportion of fatty acids which are stored in adipocytes rather than being released into the general circulation. Recent studies of chylomicron metabolism in rats highlighted adipose tissue as the most effective at trapping the fatty acids released by LPL (Hultin et al. 1996) and we would suggest that it is the ASP pathway which gives it this directionality.

If the adipocyte cannot adequately assimilate the fatty acids released from the chylomicron, then excess fatty acids will enter the general circulation (Fig. 2) and excess fatty acid entry into the general circulation will stimulate the increased secretion of VLDL particles by the liver, which will then result in an increased number of LDL particles (Sniderman et al. 1992a). The increased fatty acid flux may also contribute to the syndrome of insulin resistance, reviewed recently by Frayn et al. (1996). More to the point here is that the overproduction of hepatic apo B particles in hyperapobetalipoproteinaemia (hyperapo B) or familial combined hyperlipidaemia (FCHL) may be secondary to a fault in the peripheral metabolism of chylomicrons; i.e., a reduced rate of uptake of fatty acids by adipocytes (Sniderman et al. 1992b).

This reduced rate will also have other consequences. In vitro studies have demonstrated that increased fatty acid concentrations will inhibit the action of LPL (Bengtsson \& Olivecrona, 1980), will displace it from the endothelial surface (Saxena et al. 1989), and will separate the apo C-II-LPL complex which is essential for triacylglycerol hydrolysis (Saxena \& Goldberg, 1990). From these studies, two in vivo predictions can be made: first, if the tissue uptake of fatty acids is diminished, plasma triacylglycerol

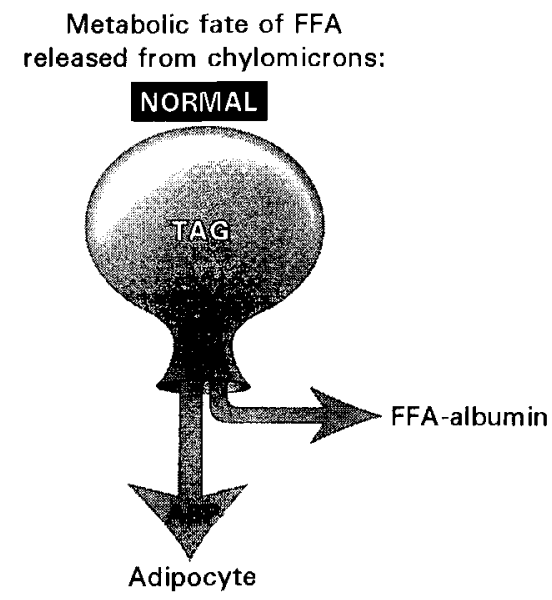

Fig. 1. The normal disposition of fatty acids released from chylomicrons: a portion escaping to the systemic circulation, a major portion being trapped by the adipocyte. Lipoprotein lipase (EC 3.1.1.34; LPL) activity is maintained by uptake, i.e. trapping of fatty acids by the adipocyte. ASP, acylation-stimulating protein; FFA, free fatty acids; TAG, triacylglycerols. 


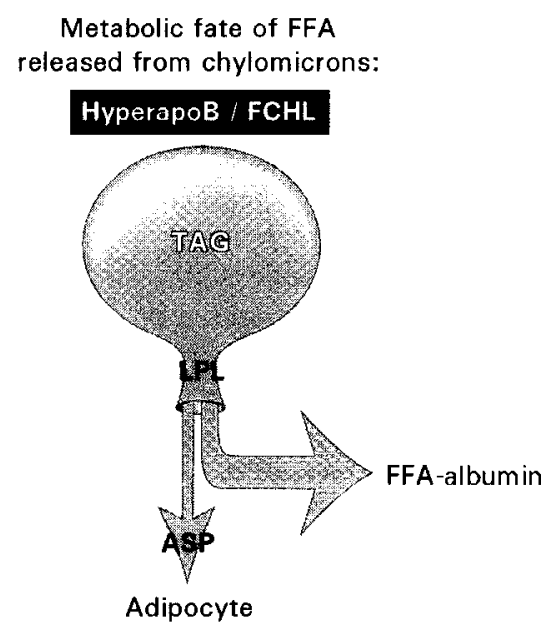

Fig. 2. The disposition of fatty acids released from chylomicrons in patients with hyperapobetalipoproteinaemia or familial combined hyperlipidaemia (hyperapo $\mathrm{B} / \mathrm{FCHL}$ ) due to a defective acylation-stimulating protein (ASP) pathway: the portion escaping to the systemic circulation being larger than normal, the portion trapped by the adipocyte being smaller than normal. Note that lipoprotein lipase $(E C$ 3.1.1.34; LPL) activity is reduced secondary to product inhibition. FFA, free fatty acids; TAG, triacylglycerols.

clearance will be delayed in the absence of any abnormality in LPL itself; second, abnormal triacylglycerol-rich chylomicron remnants will be characteristic of such a peripheral defect. Hepatic uptake of such remnants will also stimulate the secretion of VLDL particles because it represents a second mechanism of increased delivery of fatty acids to the liver (Sniderman et al. 1992a,b). Moreover, given that these triacylglycerolrich remnants accumulate in plasma, whereas normal chylomicron remnants do not, it seems likely that they are removed less readily by the liver than are the normal chylomicron remnant particles. Certainly, impaired triacylglycerol clearance is characteristic of hyperapo B or FCHL (Genest et al. 1986), as is the postprandial accumulation of larger, presumably triacylglycerol-enriched, remnants (Castro Cabezas et al. 1993). If the difference in removal rate lies primarily in the effectiveness with which normal and abnormal remnant particles are removed by the liver, it becomes unnecessary to consider the liver to be at fault in any important respect. One of the most striking, and least commented on, features of hyperapo B or FCHL is that the metabolism of all the lipoprotein classes is abnormal. If the model presented here is valid, more and more of the multiple features of these conditions can be explained by a single, critically-sited, peripheral removal defect.

\section{REDUCED LPL ACTIVITY: DELAYED PLASMA TRIACYLGLYCEROL CLEARANCE BUT NORMAL ADIPOCYTE FATTY ACID TRAPPING}

The line of reasoning can be extended: the defect must be post LPL, not only in LPL itself. If LPL itself were the problem, but the ASP pathway were normal, the rate of triacylglycerol hydrolysis would be reduced, the rate of fatty acid released would be reduced, but there would be no impairment of fatty acid uptake by the adipocytes (Fig. 3). Consequently, there would not be excess diversion of fatty acids to the liver, nor would there be the production of abnormal triacylglycerol-rich lipoproteins which would also arrive there. Since only the normal portion of exogenous fatty acids reach the liver, the secretion rate of VLDL particles would be normal. 


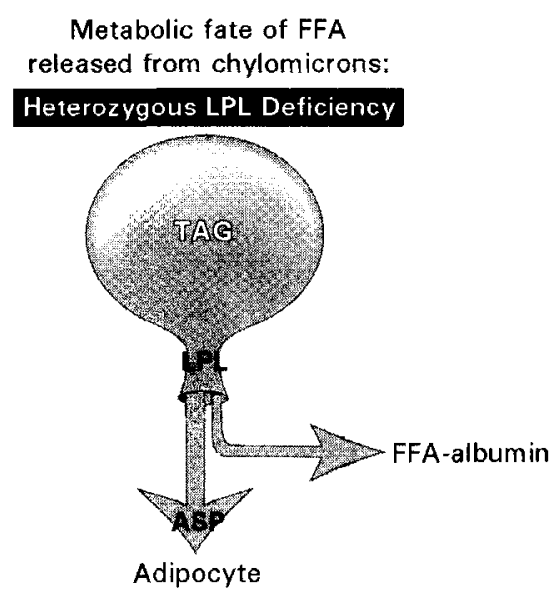

Fig. 3. The disposition of fatty acids released from chylomicrons in patients with heterozygous lipoprotein lipase (EC 3.1.1.34; LPL) deficiency. The rate of release of fatty acids is reduced because their mass is reduced but their subsequent disposition is normal because the acylation-stimulating protein (ASP) pathway is normal. Moreover, because fatty acids are trapped effectively by adipocytes, although LPL mass is much reduced, LPL activity is maintained and only modest postprandial hypertriacylglycerolaemia results. FFA, free fatty acids; TAG, triacylglycerols.

Only limited experimental evidence is available to test this model. The best human model is heterozygous LPL deficiency. Numerous molecular defects have been characterized and affected individuals can be recognized precisely. Moreover, the effects of many of these defects on LPL function have also been determined. The two commonest mutations in the Quebec population, mutation 188 and 207, both affect the catalytic site of the enzyme without affecting its mass or binding characteristics (Murthy et al. 1996). In these patients, fasting triacylglycerols are increased, as is VLDL-apo B, and plasma triacylglycerol clearance is delayed. LDL-apo B, however, is normal (Sniderman et al. 1995; Murthy et al. 1996; P. Julien, personal communication).

We interpret these results as follows: although LPL mass is normal, LPL catalytic activity is reduced by half. Thus, the rates at which chylomicrons and VLDL bound to the endothelial surface can be hydrolysed are also both markedly reduced. On the other hand, since the ASP pathway is normal, most of the fatty acids which are released will be taken up by adipocytes and consequently few will be released to the liver. Moreover, because fatty acid trapping by adipocytes is normal, capillary concentrations of fatty acids will not rise abnormally and, therefore, triacylglycerol-rich chylomicron remnants will not be detached prematurely from the endothelial surface. Because only the normal proportion of exogenous fatty acids reach the liver either as fatty acids or as triacylglycerols within chylomicron remnants, VLDL secretion is normal and that is why the LDL-apo B is normal.

\section{EVIDENCE FOR IMPAIRED TRIACYLGLYCEROL SYNTHETIC ACTIVITY AS A CAUSE OF HYPERAPOBETALIPOPROTEINAEMIA OR FAMILIAL COMBINED HYPERLIPIDAEMIA}

Two different examples will be noted: ASP-receptor-defective hyperapo B and omental obesity. In a subset of patients with hyperapo B, studies of cultured skin fibroblasts have demonstrated reduced responsiveness to ASP (Cianflone et al. 1990a). That is to say, lesser increases in triacylglycerol synthesis were induced by addition of ASP to the medium than 
were observed in similar cells obtained from normal subjects, patients with type IV hyperlipoproteinaemia with normal apo B, or patients with familial hypercholesterolaemia. Studies with radiolabelled ASP revealed evidence of high-affinity specific binding in normal subjects which was reduced in the hyperapo B patients but not in the other two patient subgroups. In such patients, reduced fatty acid uptake in vivo would be the expected consequence, with all the attendant results discussed previously. In addition, plasma ASP levels have now been shown to be elevated in patients with coronary artery disease (Cianflone et al. 1997), and from these in vivo observations has emerged the concept that this may represent an adaptation to the reduced effectiveness of ASP-receptor function which has been demonstrated in vitro.

Much interest has accompanied the hypothesis that increased release of fatty acids from adipocytes might be responsible for the increased secretion of VLDL particles in omental obesity and FCHL. At least with respect to FCHL, this seems not to be the case since reduced, rather than increased, lipolytic responses were observed in adipocytes from these patients (Reynisdottir et al. 1995). Moreover, reduced, rather than increased, amounts of hormone-sensitive lipase (EC 3.1.1.3) were also documented in this study. Fatty acid release may indeed be increased in omental adipocytes, but the rate of triacylglycerol synthesis is also reduced, just as it is in ASP-receptor-defective hyperapo B (Edens et al. 1993; Maslowska et al. 1993) and there is some evidence (although not definitive) that the reduced rate of triacylglycerol synthesis in omental obesity is related to a reduced response to ASP (Maslowska et al. 1993). If so, since it would be expected that ASP influences the rate of re-esterification, an increased rate of release of fatty acids could be the consequence of reduced ASP effect rather than increased lipolysis. Whatever the mechanism of the decreased triacylglycerol synthetic capacity, the pathophysiology of the dyslipoproteinaemia of omental obesity may be very similar to that of ASP-receptor-defective hyperapo B.

\section{SUMMARY}

Much has recently been learned about the processes involved in postprandial triacylglycerol clearance. As discussed previously, important differences in the metabolism of chylomicrons and VLDL have become apparent. The ASP pathway has also been recognized and appears to play a critical role in chylomicron metabolism. The ASP pathway is activated in order to trap the fatty acids released from chylomicrons by the action of LPL and there is now unequivocal in vivo evidence in human subjects that ASP is generated by adipocytes in the postprandial period. These findings match the in vitro data showing that chylomicrons, but not the other plasma lipoproteins or fatty acids, activate the generation of ASP by cultured human adipocytes.

An inverse relationship appears to exist between the proportion of fatty acids taken up by adipocytes and that released into the general circulation. Too great a release into the general circulation because of diminished trapping of fatty acids released from chylomicrons appears to be critical in the pathogenesis of the dyslipoproteinaemias associated with hyperapo B or FCHL and omental obesity. Evidence has been presented that dysfunction of the ASP pathway may be one of the causes of this disorder.

Put differently, the ASP pathway is essential for the normal clearance and disposition of dietary fatty acids. Binding of chylomicrons to capillary endothelium followed by lipolysis by LPL results in the sudden liberation of fatty acids, and in the marked generation of ASP by adipocytes. The ASP that is generated is essential if LPL is to continue to form fatty acids at a normal rate. It is essential also if the fatty acids which are formed are to enter the adipocyte rather than exit into the general circulation. The transport 
vehicle, the chylomicron, therefore stimulates the formation of the peptide, ASP, which is responsible for its successful metabolism. Thus, the ASP pathway provides the metabolic coordination between the chylomicron and the adipocyte, which we describe as microenvironmental metabolic regulation and which we believe is essential for the normal clearance of dietary triacylglycerol from plasma.

This research has been supported by grants from the Medical Research Council of Canada and Servier Amérique Inc.

\section{REFERENCES}

Baldo, A., Sniderman, A. D., St-Luce, S., Kohen, R., Maslowska, M., Hoang, B., Monge, J. C., Bell, A., Mulay, S. \& Cianflone, K. (1993). The adipsin-acylation stimulating protein system and regulation of intracellular triglyceride synthesis. Journal of Clinical Investigation 92, 1543-1557.

Bengtsson, G. \& Olivecrona, T. (1980). Lipoprotein lipase. Mechanism of product inhibition. European Journal of Biochemistry 106, 557-562.

Castro Cabezas, M., de Bruin, T. W., Jansen, H., Kock, L. A. W., Kortlandt, W. \& Erkelens, D. W. (1993). Impaired chylomicron remnant clearance in familial combined hyperlipidemia. Arteriosclerosis \& Thrombosis 13, 804-814.

Cianflone, K., Kalant, D., Marliss, E. B., Gougeon, R. \& Sniderman, A. D. (1995). Response of plasma ASP to a prolonged fast. International Journal of Obesity 19, 604-609.

Cianflone, K. \& Maslowska, M. (1995). Differentiation induced production of ASP in human adipocytes. European Journal of Clinical Investigation 25, 817-825.

Cianflone, K., Maslowska, M. \& Sniderman, A. D. (1990a). Impaired response of fibroblasts in patients with hyperapobetalipoproteinemia to acylation stimulating protein. Journal of Clinical Investigation 85, 722-730.

Cianflone, K., Roncari, D. A. K., Maslowska, M., Baldo, A., Forden, J. \& Sniderman, A. D. (1994). The adipsinacylation stimulating protein system in human adipocytes: Regulation of triacylglycerol synthesis. Biochemistry 33, 9489-9495.

Cianflone, K., Sniderman, A. D., Walsh, M. J., Vu, H., Gagnon, J. \& Rodriguez, M. A. (1989a). Purification and characterization of acylation-stimulating protein. Journal of Biological Chemistry 264, 426-430.

Cianflone, K., Vu, H., Walsh, M., Baldo, A. \& Sniderman, A. D. (1989b). Metabolic response of Acylation Stimulating Protein to an oral fat load. Journal of Lipid Research 30, 1727-1733.

Cianflone, K., Yasruel, Z., Rodriguez, M. A., Vas, D. \& Sniderman, A. D. (1990b). Regulation of apoB secretion from HepG2 cells: evidence for a critical role for cholesterol ester synthesis in the response to a fatty acid challenge. Journal of Lipid Research 31, 2045-2056.

Cianflone, K., Zhang, X. J., Genest, J. Jr \& Sniderman, A. D. (1997). Plasma acylation stimulating protein in coronary artery disease. Arteriosclerosis, Thrombosis \& Vascular Biology (In the Press).

Edens, N. K., Fired, S. K., Kral, J. G., Hirsch, J. \& Leibel, R. L. (1993). In vitro lipid synthesis in human adipose from three abdominal sites. American Journal of Physiology 265, E374-E379.

Frayn, K. N., Coppack, S. W., Fielding, B. A. \& Humphreys, S. M. (1994a). Coordinated regulation of hormonesensitive lipase and lipoprotein lipase in human adipose tissue in vivo: implications for the control of fat storage and fat mobilization. Advances in Enzyme Regulation 35, E308-E317.

Frayn, K. N., Shadid, S., Hamlani, R., Humphreys, S. M., Clark, M. L., Fielding, B. A., Boland, O. \& Coppack, S. W. (1994b). Regulation of fatty acid movement in human adipose tissue in the postabsorptive-topostprandial transition. American Journal of Physiology 266, E306-E317.

Frayn, K. N., Williams, C. M. \& Arner, P. (1996). Are increased plasma non-esterified fatty acid concentrations a risk marker for coronary heart disease and other chronic disease? Clinical Science 90, 243-253.

Genest, J., Sniderman, A. D., Cianflone, K., Teng, B., Wacholder, S., Marcel, Y. L. \& Kwiterovich, P. O. Jr (1986). Hyperapobetalipoproteinemia: Plasma lipoprotein responses to oral fat load. Arteriosclerosis 6, 297304.

Germinario, R., Sniderman, A. D., Manuel, S., Pratt, S., Baldo, A. \& Cianflone, K. (1993). Coordinate regulation of triacylglycerol synthesis and glucose transport by acylation stimulating protein. Metabolism 40, 574-580.

Goldberg, I. J. (1996). Lipoprotein lipase and lipolysis: central roles in lipoprotein metabolism and atherogenesis. Journal of Lipid Research 37, 693-707.

Grundy, S. M. \& Mok, H. Y. I. (1976). Chylomicron clearance in normal and hyperlipidemic man. Metabolism 25, 1225-1239.

Haagsman, H. P., de Haas, C. G. M., Geelen, M. J. H. \& van Golde, L. M. G. (1982). Regulation of triacylglycerol synthesis in the liver modulation of diacylglycerol acyltransferase activity in vitro. Journal of Biological Chemistry 257, 10593-10598. 
Hultin, M., Savonen, R. \& Olivecrona, T. (1996). Chylomicron metabolism in rats: lipolysis, recirculation of triglyceride-derived fatty acids in plasma FFA, and fate of core lipids as analyzed by compartmental modelling. Journal of Lipid Research 37, 1022-1036.

Karpe, F., Bell, M., Bjorkegren, J. \& Hamsten, A. (1995). Quantification of postprandial triglyceride-rich lipoproteins in healthy men by retinyl ester labelling and simultaneous measurement of apolipoproteins B-48 and B-100. Arteriosclerosis, Thrombosis \& Vascular Biology 15, 199-207.

Maslowska, M., Scantlebury, T., Germinario, R. \& Cianflone, K. (1997). Acute in vitro production of ASP in differentiated human adipocytes. Journal of Lipid Research 38, 1-11.

Maslowska, M., Sniderman, A. D., Maclean, L. D. \& Cianflone, K. (1993). Regional differences in triacylglycerol synthesis in adipose tissue. Journal of Lipid Research 34, 219-228.

Murthy, V., Julien, P. \& Gagne, C. (1996). Molecular pathobiology of the human lipoprotein lipase gene. Pharmacology and Therapeutics 70, 101-135.

Packard, C. J., Munro, A., Lorimer, R., Gotto, A. M. \& Shepherd, J. (1986). Metabolism of apolipoproteinB in large triglyceride-rich very low density lipoproteins in hypertriglyceridemic subjects. Journal of Clinical Investigation 77, 663-672.

Reynisdottir, S., Eriksson, M., Angelin, B. \& Arner, P. (1995). Impaired activation of adipocyte lipolysis in familial combined hyperlipidemia. Journal of Clinical Investigation 95, 2161-2169.

Saxena, U. \& Goldberg, I. J. (1990). Interaction of lipoprotein lipase with glycosaminoglycans and apolipoproteins C-II: Effects of free fatty acids. Biochimica et Biophysica Acta 1043, 161-168.

Saxena, U., Witte, L. D. \& Goldberg, I. J. (1989). Release of endothelial cell lipoprotein lipase by plasma lipoproteins and free fatty acids. Journal of Biological Chemistry 264, 4349-4355.

Scow, R. O., Blanchette-Mackie, E. J. \& Smith, L. C. (1976). Role of capillary endothelium in the clearance of chylomicrons: a model of lipid transport from blood by lateral diffusion in cell membranes. Circulation Research 39, 149-162.

Sniderman, A. D., Baldo, A. \& Cianflone, K. (1992a). The potential role of acylation stimulating protein as a determinant of plasma triglyceride clearance and intracellular triglyceride synthesis. Current Opinion in Lipidology 3, 202-207.

Sniderman, A. D., Brown, B. G., Stewart, F. F. \& Cianflone, K. (1992b). From familial combined hyperlipidemia to hyperapoB: Unravelling overproduction of hepatic apoB lipoproteins. Current Opinion in Lipidology 3, 137-142.

Sniderman, A. D., Cianflone, K. \& Eckel, R. H. (1991). Levels of acylation stimulating protein in obese women before and after moderate weight loss. International Journal of Obesity 15, 333-336.

Sniderman, A. D., Julien, P. \& Cianflone, K. (1995). Peripheral triglyceride clearance, the adipsin-ASP pathway and type IV hyperlipoproteinemia. Year Book of Endocrinology xix-xxxvii.

Tao, Y., Cianflone, K., Sniderman, A. D., Colby-Germinario, S. P. \& Germinario, R. J. (1997). Acylation stimulating protein (ASP) regulates glucose transport in the rat L6 muscle cell line. Biochimica et Biophysica Acta 1344, 221-229.

Walsh, M. J., Sniderman, A. D., Cianflone, K., Vu, H., Rodriguez, M. A. \& Forse, R. A. (1989). The effect of ASP on the adipocyte of the morbidly obese. Journal of Surgical Research 46, 470-473.

Yasruel, Z., Cianflone, K., Sniderman, A. D., Rosenbloom, M., Walsh, M. \& Rodriguez, M. A. (1991). Effect of acylation stimulating protein on the triacylglycerol synthetic pathway of human adipose tissue. Lipids 26 , $495-499$. 